This manuscript was published in Developmental Science:

Berger, P., \& Grosse Wiesmann, C. (2021). Positive emotion enhances conflict processing in preschoolers. Developmental Science.

\title{
Positive emotion enhances conflict processing in preschoolers
}

Philipp Berger ${ }^{1,2^{*}} \&$ Charlotte Grosse Wiesmann ${ }^{2}$

${ }^{1}$ Department of Neuropsychology, Max Planck Institute for Human Cognitive and Brain Sciences, Leipzig, Germany

${ }^{2}$ Research Group Milestones of Early Cognitive Development, Max Planck Institute for Human Cognitive and Brain Sciences, Leipzig, Germany

Correspondence concerning this article should be addressed to Philipp Berger, Department of Neuropsychology, Max Planck Institute for Human Cognitive and Brain Science, Stephanstraße 1a, D04103 Leipzig, Germany. Email: berger@cbs.mpg.de

\section{Data Availability}

The data that support the findings of this study are available from the corresponding author, upon reasonable request.

\section{Conflict of Interest}

We have no conflicts of interest to disclose.

\section{Acknowledgements}

We would like to thank the children and parents who volunteered to participate in the study as well as Mehves Uzun, who contributed to preparing the stimuli and acquiring the data. 


\section{Research Highlights}

- Research and theory in adults suggests that emotions can serve as a 'relevance detector' that alarms attentional systems, leading to more efficient conflict processing.

- Do preschool children show a similar facilitative effect of emotion on conflict processing, if we apply task designs analogous to adult studies showing this effect?

- We applied a modified color flanker task with a fully crossed design of conflict and emotion in preschoolers $(\mathrm{N}=43$, preregistered Bayesian sequential design).

- Our results provide indication that positive emotion can trigger efficient control processes already from early on in life. 


\begin{abstract}
The rapid detection and resolution of conflict between opposing action tendencies is crucial for our ability to engage in goal-directed behavior. Research in adults suggests that emotions can serve as a 'relevance detector' that alarms attentional and sensory systems, thereby leading to more efficient conflict processing. In contrast, previous research in children has almost exclusively stressed the impeding influence of emotion on the attentional system, as suggested by the protracted development of performance in 'hot' executive function tasks. Do preschool children show a facilitative effect of emotion on conflict processing? We addressed this question applying a modified version of a color flanker task that either involved or did not involve positive emotional stimuli in preschool children $(\mathrm{N}=43$, with preregistered Bayesian sequential design, aged $2.8-7.0$ years). Our results show a robust conflict effect with higher error rates in incongruent compared to congruent trials. Crucially, conflict resolution was faster in emotional compared to neutral conditions. Furthermore, while efficient conflict processing increases with age, we find evidence against an age-related change in the influence of positive emotion on conflict processing. Taken together, these findings provide indication that positive emotion can trigger efficient control processes already from early on in life. In contrast to the predominant view in developmental psychology, this indicates that, depending on the role that emotion has in conflict processing, emotion may show a facilitative or impeding effect already in the preschool period.
\end{abstract}

Keywords: Executive attentional control, Conflict processing, Emotion, Cognitive Control 


\section{Introduction}

Executive attentional control, the capacity of an individual to rapidly detect and resolve conflict between opposing action tendencies (Posner et al., 2009), is regarded as one of the fundamental building blocks of goal-directed and adaptive behavior (Anderson, 2002). Rapid improvements in this ability are observed in preschool age, when children start to master classical tasks of attentional control (Petersen et al., 2016), such as the Stroop (Stroop, 1935) or flanker task (Eriksen \& Eriksen, 1974). These tasks measure children's abilities to overcome distracting visual cues in favor of task-relevant cues. Besides the purely cognitive mechanisms assessed in these classical tasks, theoretical accounts in adults suggest that cognition-based conflict processing is influenced by the emotional significance of the task situation. In some of these accounts, emotions have been described as 'relevance detectors' that influence stimulus processing and behavioral control to prepare the system for action (Norman \& Shallice, 1986; Scherer, 1994), potentially facilitating conflict processing in salient situations (Kanske, 2012). This has been supported by a wide range of empirical research in adults suggesting that emotionally-valent stimuli can trigger efficient conflict processing (Kanske \& Kotz, 2011a, 2011c; Zinchenko, Obermeier, Kanske, Schröger, \& Kotz, 2017). To date, however, it is unclear when this mechanism emerges in human development, and whether conflict processing is already facilitated by emotional valence in preschool children with developing abilities of attentional control. In fact, previous research in children has almost exclusively stressed the impeding influence of emotion on the attentional system, as suggested by the protracted development of performance in 'hot' executive function tasks (Zelazo \& Carlson, 2012), which assess conflict processing in emotional situations. Thus, in the current study, we use a novel color-flanker task with a fully crossed design of conflict and emotion, investigating whether the facilitative influence of emotion on conflict processing is in place already in preschool children. 
As first described in traditional views on the interaction of emotion and cognition (Norman \& Shallice, 1986b; Scherer, 1994), salient situations may require efficient executive attentional control to ensure rapid behavioral responses, even in the face of distracting stimuli. In humans, the saliency of a situation is often indicated by emotional valence, as for example by negative, potentially threatening stimuli that bear relevance for survival (Kanske, 2012; Kanske \& Kotz, 2011b; LeDoux, 2007). At the same time, positive emotional stimuli can be seen as signals of potential reward and are thus thought to be similarly linked to the motivational approach system. Research in adults has shown that both positive and negative stimuli can trigger efficient executive attentional control. For example, in a series of studies, Kanske and colleagues (2011a, 2011b, 2011c) used a modified color flanker task in which participants had to determine the ink color of a centrally presented word, with flanker words presented in either the same (congruent) or a different (incongruent) color. In a classical flanker task, participants are typically slower and less accurate to detect a target word, when the target word is surrounded by incongruent flanker words (incongruent trials), compared to when there is no conflict between target and flanker words (congruent trials). This effect is known as the 'conflict' effect. Besides the level of conflict, Kanske and colleagues also manipulated the emotional content of the words to either emotionally-valenced or neutral words. This allowed for the assessment of conflict processing (incongruent-congruent) as a function of emotional content with a fully crossed design of conflict and emotion. The results showed that adults were faster in resolving conflict in emotional compared to neutral trials, indicating that emotion facilitated conflict processing. Notably, this facilitative effect of emotion was independent of the type of task used to assess conflict processing (i.e. flanker task or Simon task; Kanske \& Kotz, 2011b), and whether positive or negative emotions were used (Kanske \& Kotz, 2011c). Furthermore, the effect was observed in the visual and auditory modality (Kanske \& Kotz, 2011a, 2011b), as well as in the multimodal context (Zinchenko et al., 2015). In sum, these findings suggest that, in adults, the emotional salience of task stimuli might enhance and speed-up conflict processing 
(Kanske, 2012). This behavioral pattern has been suggested to be evolutionarily adaptive, as it reduces the time during which an organism is unable to respond to potentially dangerous or rewarding stimuli due to conflict (Kanske, 2012; Norman \& Shallice, 1986).

In contrast to these findings, a range of research in adults has also pointed in the opposite direction, revealing that under some circumstances emotion can significantly hamper executive attentional control (Eysenck et al., 2007; Fenske \& Eastwood, 2003; Hart et al., 2010; Kanske, 2012; Kramer et al., 2015; Lagattuta et al., 2011; Padmala et al., 2011; Pessoa et al., 2012). Some studies have argued that in certain situations, emotions might hold a distracting potential, making it more difficult to keep up goal-directed behavior (Kanske, 2012; Pessoa et al., 2012). This is underlined, for example, by studies showing that when emotional stimuli are presented before or in between conflict trials, and are thus unrelated to the actual conflict task, attentional control is usually impaired, instead of facilitated (Hart et al., 2010; Padmala et al., 2011). Further evidence for impaired performance in conflict processing caused by emotion can be found in studies in which emotion was the conflict-eliciting element in the task or, more generally, where attention to emotion was critical for task performance (Frings \& Wühr, 2012; Kramer et al., 2015; Lagattuta et al., 2011; Ochsner \& Gross, 2005; Pessoa et al., 2002). In sum, these findings in adults provide a diverse pattern of facilitating and hindering effects of emotion on conflict processing that depends on the task design and the role of emotion in the task.

In contrast to these mixed findings in adults, the prominent view in developmental psychology is that emotions tend to impair conflict processing in children. This view was established in the context of the 'hot-cold' distinction of executive control (Zelazo \& Carlson, 2012), where performance and developmental trajectories were compared between emotional ('hot') and neutral ('cold') attentional control tasks (Kramer et al., 2015; Lagattuta et al., 2011; Zelazo \& Carlson, 2012, 2020). For example, in a study by Lagatutta and colleagues (2011), performance in the Stroop-like 'Day-Night' task was compared with performance in a parallel 'hot' version of the task where emotional content was introduced. In the classical 'cold' version, children 
were shown a series of cards depicting 'day' or 'night', and were asked to respond with the respective opposite word (i.e., saying 'night' to a picture showing 'day' and vice versa). In a 'hot' version of this task, the authors manipulated the emotional valence of the stimuli by using pictures of faces showing either a happy or a sad face (i.e., 'Happy-Sad' task), and asked children to respond with the opposite emotion (i.e., saying 'happy' to a sad face and vice versa). Comparing performance in the 'cold' and 'hot' task versions showed that the emotional version elicited lower accuracy and longer response times (Kramer et al., 2015; Lagattuta et al., 2011). Drawing on a range of similar findings showing the protracted development of 'hot' executive functions (Kramer et al., 2015; Lagattuta et al., 2011; Zelazo \& Carlson, 2012), the results by Lagatutta and colleagues (2011) were interpreted as evidence that emotional content hampers conflict processing in children.

In interpreting these findings in children, however, it is important to take into account the task designs at use: Conflict was elicited by emotion and attention to emotion was critical for task performance. In fact, applying a similar task design in adults has been shown to yield the same pattern of impaired attentional control through emotional information observed in children (Kramer et al., 2015; Lagattuta et al., 2011). From these studies, therefore, it is unclear whether the facilitative effect of emotion, as previously revealed in crossed designs of conflict and emotion used in adults (Kanske, 2012), can also be found in children. An EEG study by Zinchenko and colleagues (2019) gives first indication that, using a similar task design, there may also be a facilitative effect of emotion in childhood. The authors tested 6-year-old children on a Go-NoGo task where gender of a presented face served as the Go/NoGo signal and emotional expressions were manipulated to either show emotional or neutral faces. While on the behavioral level, no influence of emotion on conflict processing was found, negative emotions were shown to modulate the magnitude of the conflict effect in event-related brain potentials (ERPs) related to conflict processing, particularly the N200 that typically has a larger amplitude for incongruent than congruent stimuli (Van Veen \& Carter, 2002). This finding 
resembles research in adults showing a similar emotion-related modulation of the N200 in early processing stages of conflict processing (Brosch et al., 2008; Kanske \& Kotz, 2011c) and therefore provides first indication that emotion may also facilitate conflict processing under certain circumstances already in childhood.

In sum, the mixed patterns of enhancing and hindering effects of emotion in adults and children raise the question whether the facilitative effect can also be observed in behavior similar to adult findings, and whether it is already present as early as the preschool period, which marks a critical take-off in executive attentional control. Do preschool children show a facilitative effect of emotion on behavioral conflict processing, if we apply task designs analogous to adult studies showing this effect?

To address these open questions, we developed an online version of a modified color flanker task for preschoolers, based on the work by Kanske and colleagues (2011) in adults. In this task, the goal of the children was to determine the color of a flower presented in the middle of the screen, while ignoring flanker flowers next to it that either had the same (congruent condition) or a different color (incongruent condition) than the central flower. The flowers were varied to either show a happy face (emotional condition) or no face (neutral condition), yielding a fully crossed design of conflict and emotion. With this design, we sought to address the open question of whether young children also showed a facilitative effect of emotion on behavioral conflict processing as identified in adults (Kanske et al., 2011a, 2011b, 2011c, 2012), or whether emotion would impede conflict processing in children as found with different tasks in childhood (Kramer et al., 2015; Lagattuta et al., 2011). In fact, although findings in adults were interpreted as an adaptive mechanism, it is unknown when and how this mechanism emerges throughout human development. In line with previous studies on the development of attentional control (Petersen et al., 2016), we expected to observe an age-related increase in conflict processing throughout the preschool period. Based on the proposal of an evolutionary adaptive mechanism, 
we hypothesized that the (facilitative) influence of emotion on conflict processing would already be present from early in life.

\section{Methods}

The current study was preregistered at: https://aspredicted.org/57ea9.pdf.

\section{Sampling approach}

We implemented a preregistered sequential Bayes Factor (BF) design (Schönbrodt et al., 2017) where data is collected until the desired level of evidence for the main hypothesis is obtained. In the Bayesian framework, the level of evidence for a hypothesis can be determined with the $\mathrm{BF}_{10}$, which quantifies how well the observed data is predicted by a hypothesis (e.g., $\mathrm{H}_{1}$ ), relative to a null- or competing hypothesis (e.g., $\mathrm{H}_{0}$ ). In general, $\mathrm{BF}_{10}$ between 1 and 3 are considered as anecdotal evidence, $\mathrm{BF}_{10}$ between 3 and 10 as moderate evidence, and $\mathrm{BF}_{10}$ above 10 as strong evidence for $\mathrm{H}_{1}$ (Lee \& Wagenmakers, 2014). Furthermore, the inverse is true for $\mathrm{H}_{0}$, with $\mathrm{BF}_{10}$ between $1 / 3$ and 1 considered as anecdotal evidence, $1 / 10$ to $1 / 3$ as moderate evidence, and $\mathrm{BF}_{10}$ under $1 / 10$ as strong evidence for $\mathrm{H}_{0}$. In a sequential $\mathrm{BF}$ design, a $\mathrm{BF}$ is prespecified as a stopping criterium at which the amount of evidence for or against the hypothesis is considered sufficient and data collection is stopped. In addition, a minimum sample size is pre-specified at which the computation of the BF is initiated for the first time to avoid false positives or negatives that might be found in small sample sizes. This allows for efficient data collection while avoiding inflated false positive rates or power loss related to sequential designs in the frequentist framework. Sequential BF designs thus allow to efficiently monitor the progression of evidence as data accumulate (Schönbrodt et al., 2017; Wagenmakers et al., 2018). A further general advantage of Bayesian designs is that they not only allow quantifying evidence for the alternative but also for the null hypothesis, thus allowing conclusions over the absence of effects beyond a null-finding. 
In the preregistration (https://aspredicted.org/57ea9.pdf), we pre-set the main parameters for our sequential BF protocol: A threshold of $\mathrm{BF} \geq 4$ for our main hypothesis (influence of emotion on conflict processing; see Statistical Analysis) was defined as a stopping criterium. This means that we would stop data collection if we observe $\mathrm{BF}_{10} \geq 4$, (indicating moderate evidence for the $\mathrm{H}_{1}$ compared to the $\mathrm{H}_{0}$ ) or $\mathrm{BF}_{10} \leq 1 / 4$ (indicating moderate evidence for $\mathrm{H}_{0}$ compared to $\mathrm{H}_{1}$ ). Furthermore, following recommendations on sequential BF designs (Schönbrodt \& Wagenmakers, 2018), we defined a minimum sample size of 30 where the analysis was initiated to avoid incidentally high BF findings at low $\mathrm{N}$. The minimum $\mathrm{N}$ and Bayes factor threshold were determined with Bayes Factor Design Analysis (Schönbrodt \& Wagenmakers, 2018) taking into account the probability of the proposed design generating misleading evidence.

\section{Participants}

The cross-sectional online study was advertised through the german platform for online-studies in children (www.kinderschaffenwissen.de), addressed to the general population, and additionally children from the institute's participant database were recruited. This resulted in a sample of $\mathrm{N}=74$ children $\left(\mathrm{M}_{\text {age }}=4.78\right.$ years, range $=2.68-8.35$ years, 43 female $)$ who started the online study, and $\mathrm{N}=43$ typically developing children between the ages of 2 and 7 years $\left(\mathrm{M}_{\mathrm{age}}=5.34\right.$ years, range $=2.84-7.01$ years, 27 female $)$ providing complete and reliable data according to our preregistered exclusion criteria (see Statistical Analysis). All children had no history of developmental disorders according to parental report. Following the sequential testing protocol, we stopped data collection after 43 complete datasets, when the desired level of evidence $\left(\mathrm{BF}_{10} \geq 4\right.$ or $\left.\mathrm{BF}_{10} \geq 1 / 4\right)$ for the main hypothesis was reached. Parental informed consent was obtained for all children before testing. The study was approved by the local ethics committee.

\section{Task Procedure}

Children participated in an online color flanker task, implemented in LabVanced (Finger et al., 2017) that we designed for tablet devices with touchscreen (see Figure 1). In this task, children 
were told a story to indicate that they will see flowers that are very thirsty and need to be watered. In order to water the flowers they need to indicate the color of the flower in the middle of the screen (blue or orange) by touching one of two corresponding buttons (left or right). Two identical flowers with either the same (congruent) or a different color (incongruent) were presented as flankers both left and right from the target. Depending on the experimental condition, the flowers either showed a happy face (emotional) or no face (neutral), yielding a fully crossed design of conflict and emotion. The control condition showed flower seeds, which were visually matched to the emotional condition. This was done to ensure that the control condition was considered 'neutral' consistently across participants as neutral faces may be attributed different emotional valence by different participants (Lee et al., 2008). In an instruction phase, parents read out standardized instructions to their children which were presented on the top of the screen. The instruction phase included a detailed explanation of the goal of the task and several tests of task understanding, including test trials indicating whether children were able to i) tell the difference between the colors and ii) understand the concept of the 'middle flower'. Furthermore, children received four practice trials with feedback in which each condition was presented once. In each of the above practice trials and the following test phase, parents were instructed not to influence children's responses. In the test phase, every child was presented with a total of 16 trials from each of the four conditions in pseudorandomized order, yielding a total of 64 trials (similar to previous studies with children, e.g. Akshoomoff et al., 2014; McDermott et al., 2007; Weintraub et al., 2013). All trials began with a $1500 \mathrm{~ms}$ preparation interval depicting a star in the middle of the screen and the response buttons in the respective positions. Then, the stimulus was presented for $1000 \mathrm{~ms}$, additionally starting the response phase, which had a variable maximum duration ranging from $2500 \mathrm{~ms}$ to $3500 \mathrm{~ms}$. This was done to introduce time pressure to speed up responses (Diederich, 2008; Diederich \& Busemeyer, 2006; Link, 1971). Each trial closed with an interstimulus interval (ISI) of $2000 \mathrm{~ms}$ depicting a turning star to capture attention in the middle of the screen. Overall, 
completion of the experiment took about 7 minutes. Reaction times (RTs) and accuracy data were collected in the course of the experiment. To ensure that children, and not adults, engaged in the task we collected audio recordings during test trials, and recorded children saying their name in the instruction phase.

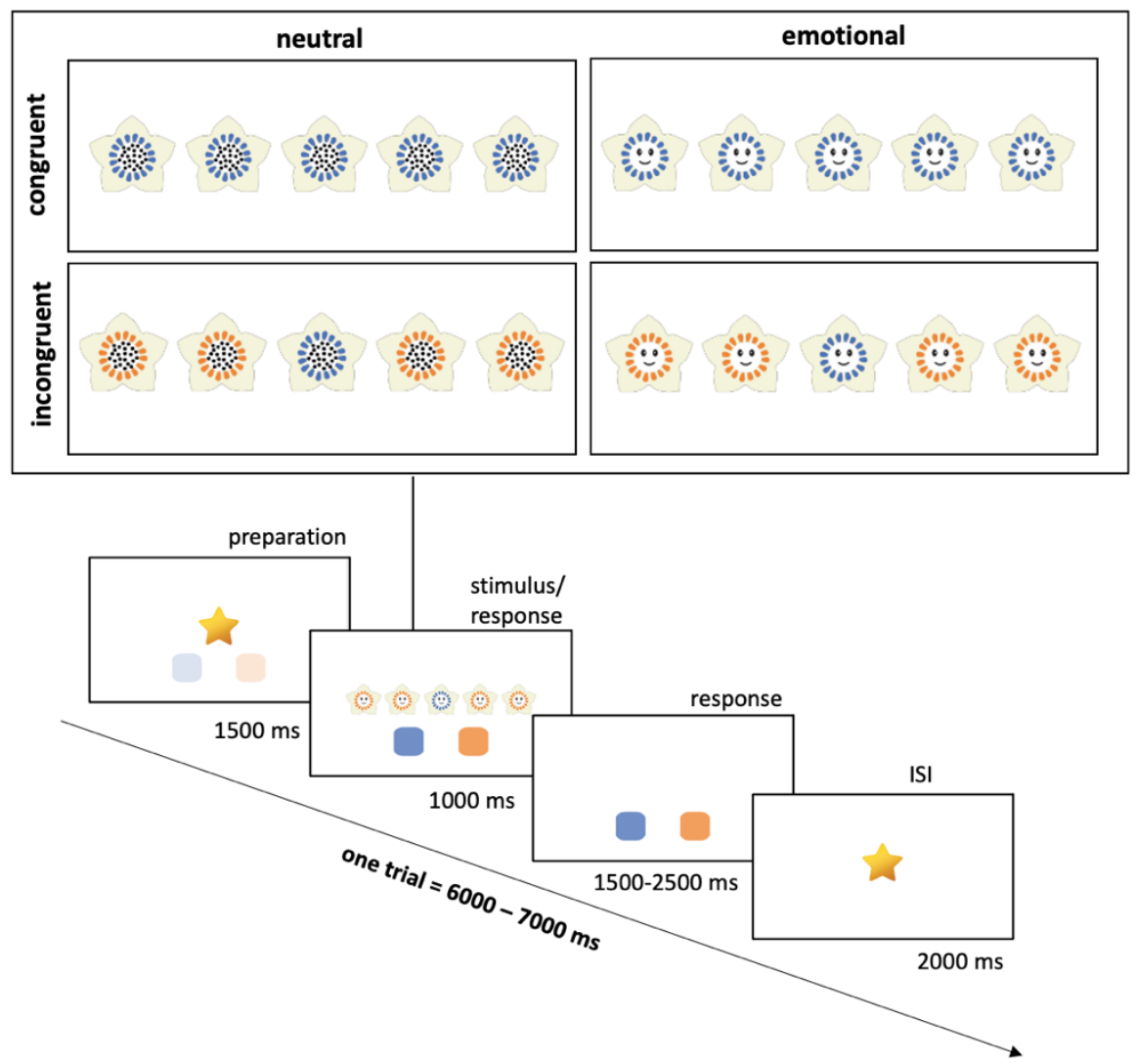

Figure 1. Modified color flanker task for preschoolers used in the current study. The task was designed for tablet devices with a touchscreen. The participant's task was to touch the color of the middle flower, ignoring that of the flanker colors (congruent vs. incongruent conditions). Flowers either showed a face (emotional condition) or no face (neutral condition), yielding a fully crossed design of conflict and emotion.

\section{Statistical Analysis}

We preprocessed and analyzed the data using R (R Core Team, 2019) and JASP (JASP Team, 2020). As defined in the preregistration, mean RTs and the average percentage of correct responses (accuracy) were calculated within each condition as an indicator of performance. 
Data was cleaned for outliers by excluding reaction times that were faster than $200 \mathrm{~ms}$ after target onset (Schmiedek et al., 2007). Trials without responses were flagged as 'false' responses and coded as the maximum RT in the respective trial. Participants were excluded if they had more than $50 \%$ trials without responses over all conditions, or more than $75 \%$ trials without responses within one of the conditions $\left(\mathrm{N}=30\right.$, median $_{\mathrm{age}}=3.95$ years, range $=2.68-8.35$ years, 15 female). Due to the challenges of obtaining high-quality data from children in online task settings (Zaadnoordijk et al., 2021), we decided to lower the preregistered threshold (50\% within each condition), in order to achieve an acceptable inclusion rate. Additionally, following the preregistration, participants were excluded if their overall accuracy was not significantly above chance according to a binomial test $(\mathrm{N}=1$, age $=3.36$ years, female).

In the resulting dataset, we derived conflict scores by subtracting performance in congruent and incongruent conditions, divided by the mean RT or accuracy of the participant (see Figure 3). The influence of emotion was determined by an emotion score, in which the conflict scores in neutral and emotional conditions were subtracted (see Figure 4). As a stopping criterium in the sequential BF protocol, we computed the BF for a direct comparison of conflict scores in neutral and emotional conditions using one-tailed Bayesian paired t-tests with a default prior described by a Cauchy distribution centered around 0 and with a width parameter of 0.35 (van Doorn et al., 2020), according to our preregistered hypotheses. This corresponds to a probability of $80 \%$ that the standardized effect size (d) lies between 0 and 1.1, based on previous literature in adults and children (Kanske \& Kotz, 2011a; McDermott et al., 2007). Once the stopping criterium of $\mathrm{BF}=4$ for this analysis was reached, Bayesian analyses were computed as preregistered. In addition, for purposes of comparability to previous studies, we calculated standard frequentist 2 (conflict) x 2 (emotion) repeated-measures ANOVAs to indicate the main effect of conflict and emotion, as well as the interaction of conflict and emotion. Furthermore, to indicate whether age was associated with task performance, we computed correlations of conflict and emotion scores with age with Bayesian statistics using default priors as preregistered. 


\section{Results}

Bayesian Sequential Testing: Preregistered Stopping Criterium

Following the preregistered sequential BF protocol, we calculated Bayesian paired t-tests comparing conflict effects in emotional and neutral conditions, starting from a minimum sample size of $\mathrm{N}=30$. After $\mathrm{N}=43$ participants $\left(\mathrm{M}_{\mathrm{age}}=5.34\right.$ years, range $=2.84-7.01$ years, 27 female $)$ the Bayesian paired t-test converged showing moderate evidence for a difference between conflict scores in $\mathrm{RT}\left(\mathrm{BF}_{10}=4.99, \mathrm{~d}=0.29,95 \%\right.$ HDI $\left.[0.05,0.60]\right)$, with higher conflict scores in the neutral compared to the emotional condition. That is, that children showed slower conflict processing in the neutral compared to the emotional condition. Robustness analyses show that the evidence is robust across a wide range of prior specifications (see Figure S1a). In the accuracy data, there was no difference in the conflict scores between the neutral and emotional condition $\left(\mathrm{BF}_{10}=0.18, \mathrm{~d}=0.06,95 \%\right.$ HDI $[0.00,0.24]$; Figure $\left.\mathrm{S} 1 \mathrm{~b}\right)$.

\section{Conflict and Emotion Effects}

For purposes of comparison with frequentist statistics, we calculated repeated-measures ANOVAs to test for main effects of conflict and emotion as well as the interaction of conflict and emotion in the RT and accuracy data. In line with the results of the Bayesian t-test, the RT data showed no main effects of conflict $\left(\mathrm{F}[1,42]=0.38, \mathrm{p}=0.54, \mathrm{BF}_{\text {incl }}=0.21, \eta_{\mathrm{p}}{ }^{2}<0.001\right)$ or emotion $\left(F[1,42]=0.4, p=0.53, B F_{\text {incl }}=0.20, \eta_{p}{ }^{2}=0.009\right)$, but a significant conflict $x$ emotion interaction $\left(\mathrm{F}[1,42]=5.41, \mathrm{p}=0.025, \mathrm{BF}_{\text {incl }}=1.49, \eta_{\mathrm{p}}{ }^{2}=0.114\right.$; see Figure $\left.2 \mathrm{a}\right)$. There was a significant conflict effect in neutral $\left(\mathrm{t}[42]=1.92, \mathrm{p}=0.031, \mathrm{BF}_{10}=2.45\right.$, one-sided), but not in emotional trials $\left(\mathrm{t}[42]=-1.21, \mathrm{p}=0.883, \mathrm{BF}_{10}=0.16\right.$, one-sided). In the accuracy data, the repeated-measures ANOVA showed a significant main effect of conflict $(F[1,42]=13.89, p<$ $\left.0.001, \mathrm{BF}_{\text {incl }}=280.30, \eta_{\mathrm{p}}{ }^{2}=0.249\right)$, with no main effect of emotion $(\mathrm{F}[1,42]=0.04, \mathrm{p}=0.84$, $\left.\mathrm{BF}_{\text {incl }}=0.17, \eta_{\mathrm{p}}^{2}<0.001\right)$ or conflict $\mathrm{x}$ emotion interaction $\left(\mathrm{F}[1,42]=0.56, \mathrm{p}=0.46, \mathrm{BF}_{\text {incl }}=\right.$ $0.25, \eta_{\mathrm{p}}{ }^{2}=0.013$; see Figure $2 \mathrm{~b}$ ), in line with the Bayesian t-test. 


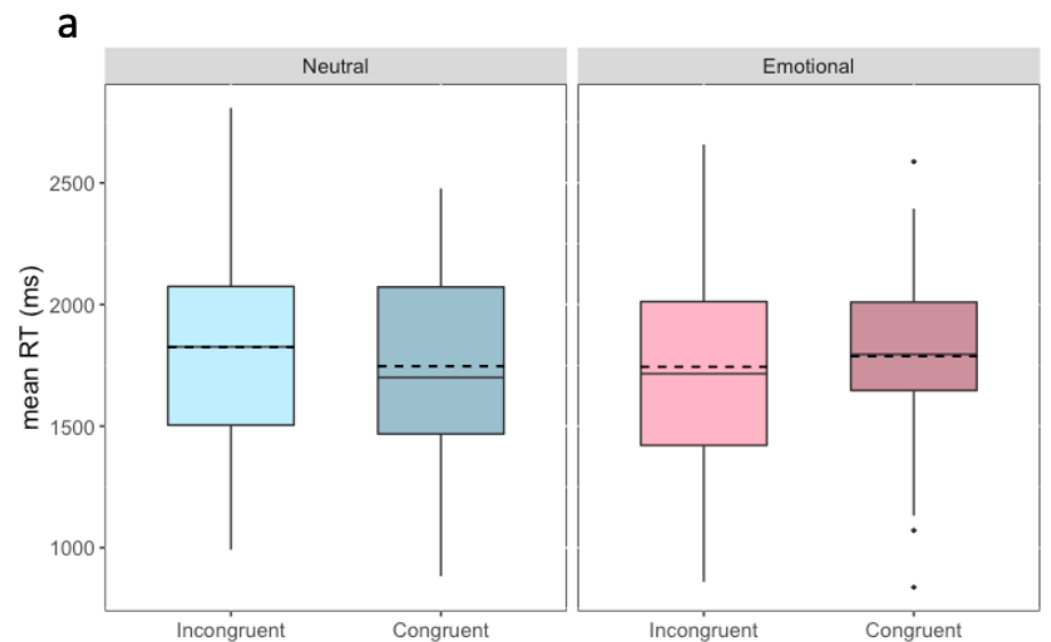

b

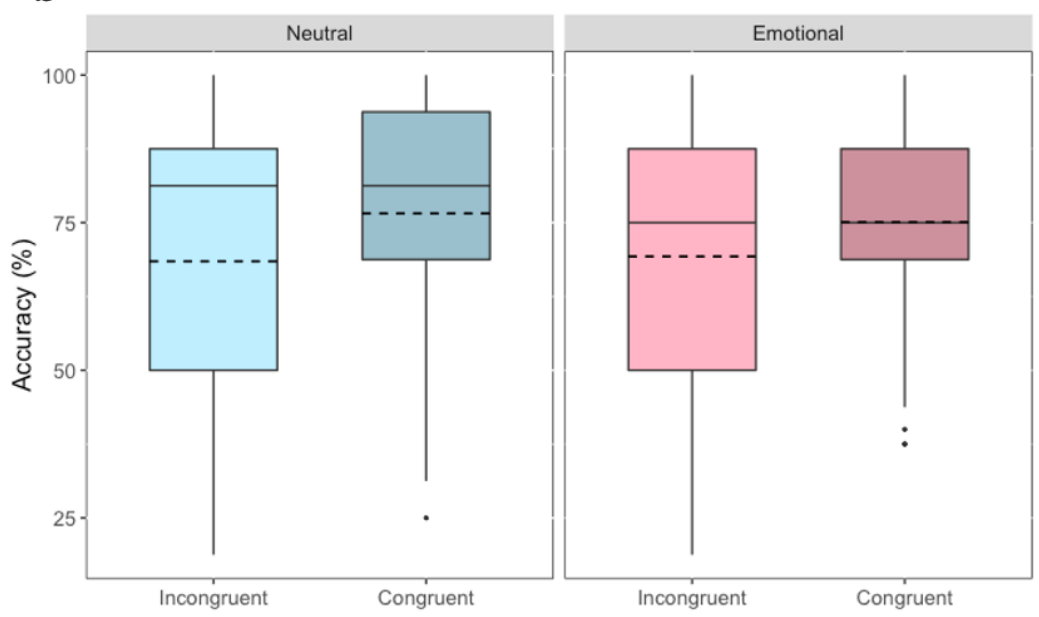

Figure 2. Box plots depicting mean reaction times (RT, a) and accuracy data (b) for emotional (red) and neutral (blue) conditions, by incongruent and congruent conditions. Results show a modulation of the conflict effect by emotional context reflected in an interaction of congruency and emotion in the RT data (a). In the accuracy data (b), a main effect of conflict reflected in lower accuracy in the incongruent compared to congruent condition was found (b). The box plots indicate the median together with the first and third quartile. In addition, the mean is shown as a dashed line.

\section{Correlation with Age}

Concerning the relation with age, we found substantial evidence for a negative correlation between age and conflict effects in the accuracy data $\left(\mathrm{BF}_{10}=32.55\right.$, Spearman's $\rho=-0.42, \mathrm{p}=$ 0.005; Figure 3b). That is, younger children showed a stronger conflict effect than older children, in line with previous literature that conflict processing improves with age (Petersen et 
al., 2016). In the RTs, we found no evidence for a correlation between age and conflict effects $\left(\mathrm{BF}_{10}=0.50\right.$, Spearman's $\rho=-0.25, \mathrm{p}=0.107$; Figure 3a). Concerning the modulation of the conflict effect by emotion, in contrast, we found evidence against a correlation with age in the RT $\left(\mathrm{BF}_{10}=0.21\right.$, Spearman's $\rho=0.04, \mathrm{p}=0.798$; Figure 4a) and no evidence for a correlation with age in the accuracy data $\left(\mathrm{BF}_{10}=0.70\right.$, Spearman's $\rho=0.15, \mathrm{p}=0.33$; Figure $\left.4 \mathrm{~b}\right)$.
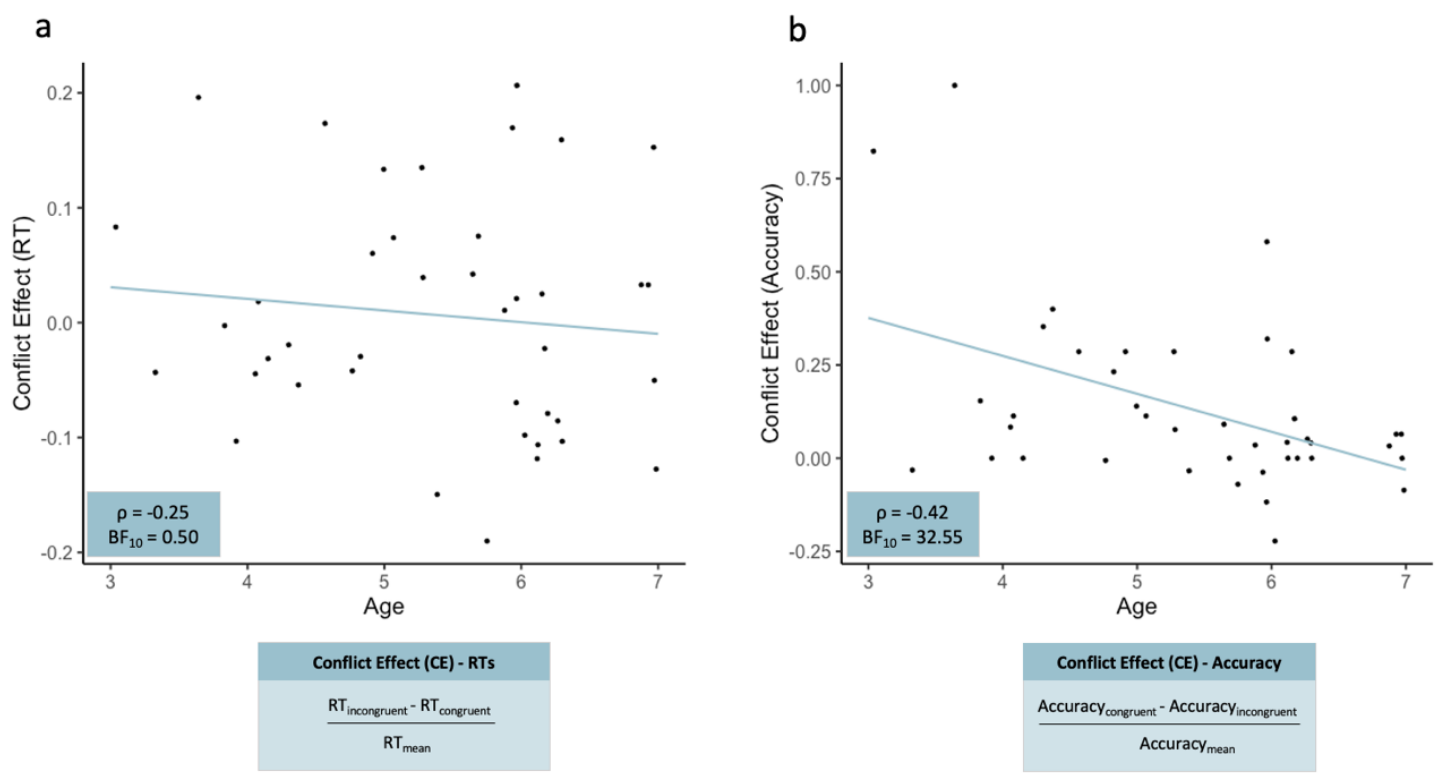

Figure 3. Scatter plots depicting correlations of children's age with conflict effects (CE) in reaction time (RT; a) and accuracy data (b). Results show substantial evidence for a correlation between age and CEs in accuracy, and a similar tendency in the RT data. CEs were calculated by subtracting performance in congruent and incongruent conditions, and dividing by the mean RT or accuracy of the participant. 

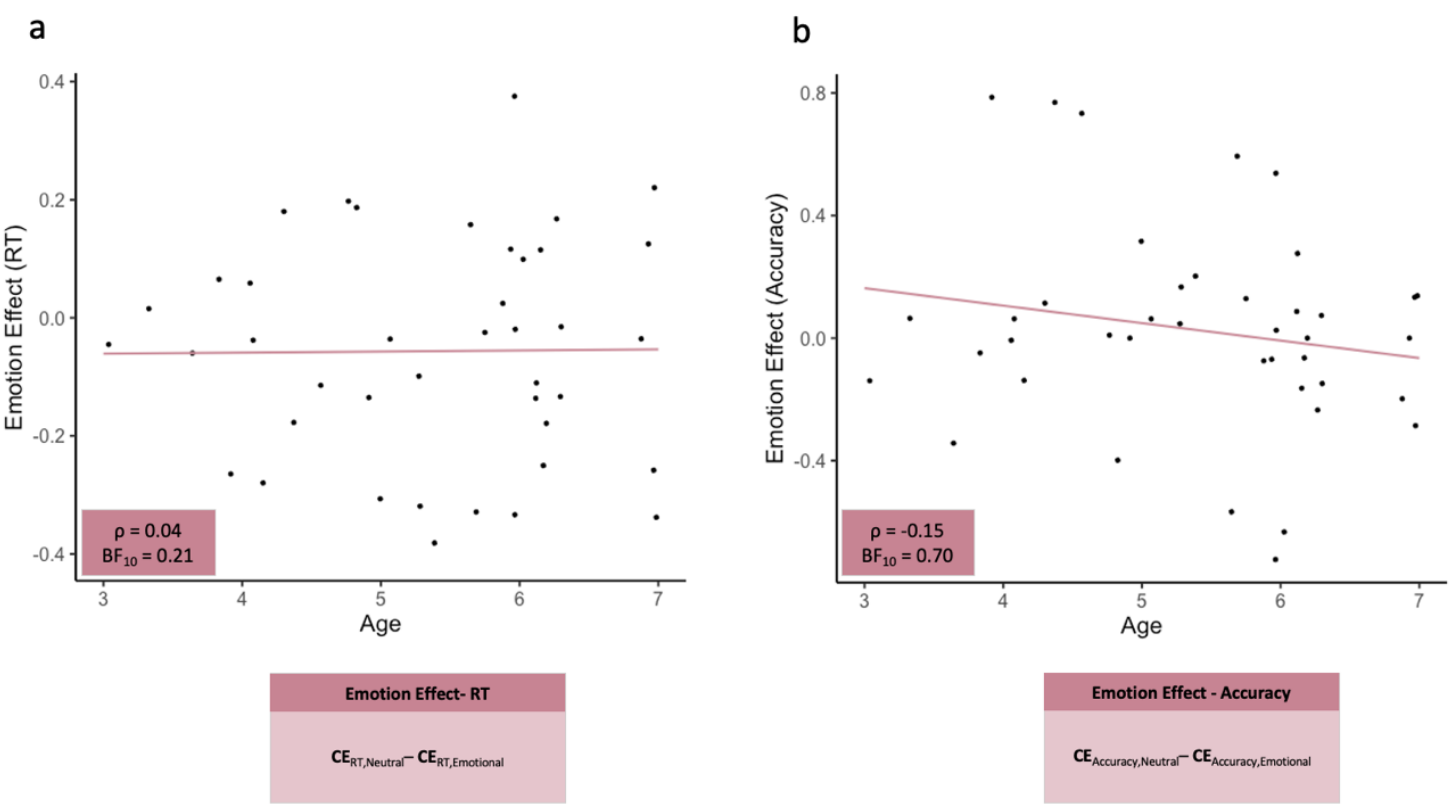

Figure 4. Scatter plots depicting correlations of children's age with emotion effects in reaction time (RT; a) and accuracy data (b). Emotion effects were calculated by subtracting conflict effects (CEs) in neutral and emotional conditions. Results show evidence against a correlation of emotion effects in RT with children's age.

\section{Discussion}

This preregistered study aimed to clarify whether emotion can facilitate attentional control in young children, and how this effect of emotion develops in preschool age, a period with extensive improvements in conflict processing. Using a modified color flanker task with a fully crossed design of conflict and emotion, our results provide evidence that positive emotional content can lead to more effective conflict processing in preschoolers. These results resemble research findings in adults (Kanske, 2012), which have been interpreted as an evolutionary adaptive mechanism in humans (Scherer, 1994). The observed facilitative effect of emotion, however, is in contrast with current views in developmental psychology that have emphasized an impeding effect of emotion on conflict processing.

The present data allow addressing a long-standing open question on the influence of emotion on cognitive processing in young children (Zelazo \& Carlson, 2012). Particularly, while 
research in adults has shown that under various task conditions, emotion can have a facilitative effect on conflict processing (Kanske \& Kotz, 2011a, 2011b, 2011c), findings in children mostly indicate that emotional content impairs conflict processing (Kramer et al., 2015; Lagattuta et al., 2011; Zelazo \& Carlson, 2012). In this study, we replicate previous findings showing a linear increase of conflict processing in the preschool period (Petersen et al., 2016). Importantly, however, our data show that preschool children demonstrate faster conflict processing for emotional compared to neutral stimuli, indicating a facilitative effect of emotion on conflict processing in preschool children, similar to the effect found in adults. These results line up with recent evidence from an EEG study conducted in 6-year-old children showing that emotions modulate the magnitude of the conflict effect observed in ERPs related to conflict processing, particularly the N200 (Zinchenko et al., 2019), although this study did not find a modulation of behavioral conflict processing. Our study thus provides the first evidence for a facilitation of conflict processing on the behavioral level in children, as early as from preschoolage.

Our findings are in line with the interpretation of a facilitative effect of emotion as an evolutionary adaptive mechanism, previously suggested in the adult literature (Kanske, 2012; Scherer, 1994). That is, emotional stimuli might signal saliency and reduce the time that an organism is incapable of responding to a potentially dangerous or reward-signaling stimulus (Kanske, 2012; Kanske \& Kotz, 2011c). Positive emotions have been shown to particularly modulate the early stages of spatial orientation (Brosch et al., 2008) and attentional control (Kanske \& Kotz, 2011c), suggesting a rapid recruitment of the attentional system. This idea is further underlined by research showing that an attentional bias towards emotional stimuli is manifested already during early infancy (Peltola et al., 2009; Vaish et al., 2008). While we found that attentional control increased in the preschool period, the facilitating influence of emotion on conflict processing in the RT data remained stable across age. These findings 
suggest that emotions modulate cognitive processing already from early childhood in line with an adaptive mechanism present early in life.

Notably, however, our results also stand in contrast to previous findings and views in developmental psychology highlighting the relatively protracted development of emotional ('hot') executive functioning (Kramer et al., 2015; Lagattuta et al., 2011; Zelazo \& Carlson, 2012). This discrepancy might be explained by the role that emotion has in the different task designs. In particular, we suggest that there may be two dimensions that determine the effect that emotion has on conflict processing. One dimension is whether the conflict itself requires overcoming emotions or not (Frings \& Wühr, 2012; Kramer et al., 2015; Ochsner \& Gross, 2005; Pessoa et al., 2002). So far, studies in children have predominantly used tasks in which emotion is critical for the conflict task: Parallel 'hot' versions of classical 'cold' executive functioning tasks, such as the 'Happy-Sad task' (Kramer et al., 2015; Lagattuta et al., 2011) elicit conflict between emotional responses, which has been referred to as 'emotional conflict' (Egner et al., 2008; Etkin et al., 2006). In these tasks, children have to attend to and overcome the emotion in order to respond correctly. Thereby, the emotion contributes to and increases the saliency of the conflict, potentially making it more difficult for children to process and overcome this conflict. Studies in children and adults that found a facilitative influence of emotion on conflict processing, in contrast, have used task designs in which emotion was a feature of the stimuli and in which emotion did not constitute the conflict to be resolved (Kanske, 2012; Zinchenko et al., 2019). More specifically, in order to respond correctly in the modified color flanker task used in adults (Kanske, 2012) and in our study, participants did not have to focus on the emotional information, but the conflict was created by other features of the stimuli (i.e., their color). In this case, increasing the saliency of the stimuli through their emotional content would facilitate focusing on the central stimulus and ignoring the flanker stimuli, making it easier to overcome the color conflict between them. In contrast to the tasks frequently used in children, where emotion increased the saliency of the conflict impeding 
conflict processing (Kramer et al., 2015; Lagattuta et al., 2011; Zelazo \& Carlson, 2012), these tasks therefore show a facilitative effect of emotion on conflict processing. The other dimension to be considered is whether emotions are included within the task itself, thus increasing the stimulus saliency, or whether emotions are induced independently of the task (Kanske, 2012). That is, in studies showing a facilitative effect of emotion on conflict processing, the emotional stimuli are typically embedded in the task as stimuli, such that participants need to process these stimuli in order to solve the task (Kanske, 2012; Kanske \& Kotz, 2011a, 2011c; Zinchenko et al., 2019). This contrasts with studies that modulate or induce emotion through the presentation of additional procedures unrelated to the conflict task itself, such as through presenting emotional stimuli before (e.g., Hart et al., 2010) or in between conflict trials (e.g., Padmala et al., 2011), or by inducing emotions in the participants (Eysenck et al., 2007). In these cases, the induced emotional state has been found to hamper conflict processing, in line with a distracting effect of task-irrelevant emotions (Kanske, 2012).

In the current study, we used a task in which emotion does not create the conflict itself but is embedded in the task, and correspondingly found a facilitating effect of emotions. These findings go beyond previous studies by showing that this facilitative effect is already in place in the early preschool period, and is also displayed in behavior. Following previous research and theory in adults (Frings \& Wühr, 2012; Kanske, 2012; Ochsner \& Gross, 2005; Pessoa et al., 2002; Zinchenko, Obermeier, Kanske, Schröger, \& Kotz, 2017), we therefore suggest that depending on the function emotion has in the actual conflict, it has an impeding or a facilitative effect on conflict processing, and that these different mechanisms may already be in place, similar to the ones in adults, at the emergence of conflict processing in the early preschool years.

This view is also in line with evidence from a study that compared a classical 'cold' attentional control task, i.e. the 'Day-Night' task, with variations in which emotion did not create the conflict, but was embedded in the task as stimuli. One such variation is the 'Boy-Girl' task in 
which children are shown photographs of emotion faces and are asked to respond with the opposite gender. In line with our suggestion, children made more errors and took longer to respond in the 'Happy-Sad' task where the emotion was the conflict-eliciting element compared to the 'Boy-Girl' task where the emotional faces were solely an attribute of the stimuli. Moreover, the 'Boy-Girl' variation was easier for the children than the classical 'Day-Night' task (Kramer et al., 2015). These results in children support the view that the particular influence of emotion depends on whether the emotional content of the stimuli creates the conflict or whether it is an additional feature of the stimuli that is independent of the actual conflict. Such indirect comparisons of performance in different tasks, as between the variations of the 'Day-Night' task by Kramer and colleagues (2015), however, also offer a number of alternative explanations. Particularly, Kramer and colleagues (2015) discuss that the 'Boy-Girl' variation might have been easier for children due to ample experience categorizing people by gender, or simpler discrimination due to visual features of photographs compared to pictograms in the 'Day-Night' and 'Happy-Sad' task. In our study, in contrast, we manipulated emotion directly within the same task in a fully-crossed design of conflict and emotion. Our task therefore allows us to exclude such alternative explanations related to task or stimulus differences.

With this new task, our study opens up new avenues for future developmental research on the role of emotion in conflict processing. An open question is whether similar effects are found using different task conditions (Kanske \& Kotz, 2011a, 2011b, 2011c) and stimulus modalities, as found in the literature on adults (Kanske, 2012; Kanske \& Kotz, 2011c) and older adults (Zinchenko, Obermeier, Kanske, Schröger, Villringer, et al., 2017; Zinchenko et al., 2018). Future studies should investigate in more detail how types of emotion and the way emotion are conveyed by the stimuli influence the facilitative effect on conflict processing in preschoolers. In particular, since the current study is focused on positive emotions, an open question is whether the facilitative effect is independent of emotional valence and is present also for 
negative emotions, as has been shown in adults (Kanske \& Kotz, 2011a, 2011c, 2011d). Furthermore, the current study uses simple smiley faces to convey emotional content in the task. Although previous studies suggest similar effects for more naturalistic stimuli (i.e., photographs; Kramer et al., 2015) and across a number of different task types (Kanske \& Kotz, 2011c), it remains to be investigated whether the facilitative effect of emotion in preschoolers generalizes across design features. Together, this would strengthen the interpretation of a supramodal facilitative effect on attentional control as an adaptive mechanism that is already present in preschool children and might give an indication how this effect varies as a function of emotion. Moreover, future research may follow up on whether social contents that are not specifically emotional can also facilitate conflict processing. In this context, it would further be interesting to know whether the effect is observable already in children younger than 3 years of age, as precursory skills of attentional control have been shown to develop already in infancy (Fiske et al., 2021; Fiske \& Holmboe, 2019). Notably, however, in view of the relatively high exclusion rate in young preschool children with the current task (see Statistical Analysis and Table S1), a new task would need to be developed to test younger children. Further, in adults, there is indirect evidence that emotion might modulate early processing stages of conflict processing, indicating that already the detection of conflict may be affected by emotional stimuli (Brosch et al., 2008; Kanske \& Kotz, 2011c). In preschool children, it remains an open question whether emotions target the process of conflict detection or resolution. In fact, a further possibility to interpret the results of the current study is that children might have been more engaged in performing the conflict task in the case of positive emotional compared to neutral stimuli, rather than the emotion facilitating the conflict processing itself. The findings that an ERP component associated with early stages of conflict processing, such as the N200 (Van Veen \& Carter, 2002) is modulated by emotion in similar task contexts in older children and adults (Kanske \& Kotz, 2011c; Zinchenko et al., 2019), however, suggests that a facilitative effect of emotion on early processing components is the more parsimonious interpretation of 
our findings. This could be followed up with a similar EEG experiment in young preschoolers in the age range of the current study. Relatedly, it remains an open question which brain structures might underlie the modulatory influence of emotion on conflict processing in children. Despite a large body of literature showing the influence of a cognitive control network, including prefrontal and parietal cortices, for conflict (Niendam et al., 2012), the ventral anterior cingulate cortex has been identified as a key structure underlying the facilitative effect of emotion on conflict processing in adults (Kanske \& Kotz, 2011b). In children, first evidence suggests that the maturation of distinct components of the adult cognitive control network plays an important role in the early development of conflict processing that involves overcoming emotions (usually referred to as 'hot') compared to tasks that do not involve emotion ('cold'; Berger et al., preprint). However, it remains unclear to what extent the maturation of those brain structures, and particularly the ventral anterior cingulate cortex might be related to the modulatory effect of emotion on conflict processing in early childhood. This question might be addressed by relating behavioral changes in the way emotion influences conflict processing in young children, as captured in our task, to brain maturation using structural Magnetic Resonance Imaging techniques. Finally, an important endeavor of future research will be to investigate whether children with developmental disorders closely related to impairments in executive functioning, such as attention deficit/hyperactivity or conduct disorder, show differences in the way emotion influences conflict processing.

\section{Conclusions}

The present preregistered study provides evidence that, from early on in childhood, as abilities of attentional control emerge, emotional stimuli can facilitate conflict processing. While attentional control improved with age, the influence of emotion remained constant from at least 3 years of age. In contrast to previous findings and theories in developmental psychology, these results indicate that emotion can trigger efficient control processes already from early on in life. 
We conclude that the influence of emotion on conflict processing in children, similar to adults, is not determined by the presence or absence of emotions, but rather that emotion may increase stimulus saliency and its effect on conflict processing may thus depend on the role emotion plays in the conflict. 


\section{References}

Akshoomoff, N., Newman, E., Thompson, W. K., McCabe, C., Bloss, C. S., Chang, L., Amaral, D. G., Casey, B., Ernst, T. M., Frazier, J. A., \& others. (2014). The NIH Toolbox Cognition Battery: Results from a large normative developmental sample (PING). Neuropsychology, 28(1), 1.

Anderson, P. (2002). Assessment and development of executive function (EF) during childhood. Child Neuropsychology, 8(2), 71-82. https://doi.org/10.1076/chin.8.2.71.8724

Berger, P., Friederici, A. D., \& Wiesmann, C. G. (2021). Maturation of distinct neural components of the cognitive control network support early development of inhibitory control. Preprint (BioRxiv). https://doi.org/10.1101/2021.07.02.450852

Brosch, T., Sander, D., Pourtois, G., \& Scherer, K. R. (2008). Beyond fear: Rapid spatial orienting toward positive emotional stimuli: Research article. Psychological Science, 19(4), 362-370. https://doi.org/10.1111/j.1467-9280.2008.02094.x

Diederich, A. (2008). A further test of sequential-sampling models that account for payoff effects on response bias in perceptual decision tasks. Perception \& Psychophysics, 70(2), 229-256.

Diederich, A., \& Busemeyer, J. R. (2006). Modeling the effects of payoff on response bias in a perceptual discrimination task: Bound-change, drift-rate-change, or two-stage-processing hypothesis. Perception \& Psychophysics, 68(2), 194-207.

Egner, T., Etkin, A., Gale, S., \& Hirsch, J. (2008). Dissociable neural systems resolve conflict from emotional versus nonemotional distracters. Cerebral Cortex, 18(6), 1475-1484.

Eriksen, B. A., \& Eriksen, C. W. (1974). Effects of noise letters upon the identification of a target letter in a nonsearch task. Perception \& Psychophysics, 16(1), 143-149. https://doi.org/10.3758/BF03203267

Etkin, A., Egner, T., Peraza, D. M., Kandel, E. R., \& Hirsch, J. (2006). Resolving Emotional Conflict: A Role for the Rostral Anterior Cingulate Cortex in Modulating Activity in the Amygdala. Neuron, 51(6), 871-882. https://doi.org/10.1016/j.neuron.2006.07.029

Eysenck, M. W., Derakshan, N., Santos, R., \& Calvo, M. G. (2007). Anxiety and cognitive performance: Attentional control theory. Emotion, 7(2), 336.

Fenske, M. J., \& Eastwood, J. D. (2003). Modulation of focused attention by faces expressing emotion: Evidence from flanker tasks. Emotion, 3(4), 327.

Finger, H., Goeke, C., Diekamp, D., Standvoß, K., \& König, P. (2017). LabVanced: A Unified JavaScript Framework for Online Studies. Computational Social Science IC, 2(2). https://doi.org/10.1126/science.aac4716

Fiske, A., de Klerk, C., Lui, K. Y., Collins-Jones, L. H., Hendry, A., Greenhalgh, I., Hall, A., Scerif, G., Dvergsdal, H., \& Holmboe, K. (2021). The neural correlates of inhibitory control in 10-month-old infants: A functional near-infrared spectroscopy study.

Fiske, A., \& Holmboe, K. (2019). Neural substrates of early executive function development. Developmental Review, 52, 42-62.

Frings, C., \& Wühr, P. (2012). Don't be afraid of irrelevant words: The emotional Stroop effect is confined to attended words. Cognition \& Emotion, 26(6), 1056-1068.

Hart, S. J., Green, S. R., Casp, M., \& Belger, A. (2010). Emotional priming effects during Stroop task performance. Neuroimage, 49(3), 2662-2670. 
JASP Team. (2020). JASP (Version 0.14.1)[Computer software]. https://jasp-stats.org/

Kanske, P. (2012). On the influence of emotion on conflict processing. Frontiers in Integrative Neuroscience, 6(JULY 2012), 42. https://doi.org/10.3389/fnint.2012.00042

Kanske, P., \& Kotz, S. A. (2011a). Emotion triggers executive attention: Anterior cingulate cortex and amygdala responses to emotional words in a conflict task. Human Brain Mapping, 32(2), 198-208. https://doi.org/10.1002/hbm.21012

Kanske, P., \& Kotz, S. A. (2011b). Emotion triggers executive attention: Anterior cingulate cortex and amygdala responses to emotional words in a conflict task. Human Brain Mapping, 32(2), 198-208. https://doi.org/10.1002/hbm.21012

Kanske, P., \& Kotz, S. A. (2011c). Positive emotion speeds up conflict processing: ERP responses in an auditory Simon task. Biological Psychology, 87(1), 122-127. https://doi.org/10.1016/j.biopsycho.2011.02.018

Kanske, P., \& Kotz, S. A. (2011d). Conflict processing is modulated by positive emotion: ERP data from a flanker task. Behavioural Brain Research, 219(2), 382-386. https://doi.org/10.1016/j.bbr.2011.01.043

Kramer, H. J., Lagattuta, K. H. ansen, \& Sayfan, L. (2015). Why is happy-sad more difficult? Focal emotional information impairs inhibitory control in children and adults. Emotion (Washington, D.C.), 15(1), 61-72. https://doi.org/10.1037/emo0000023

Lagattuta, K. H., Sayfan, L., \& Monsour, M. (2011). A new measure for assessing executive function across a wide age range: Children and adults find happy-sad more difficult than day-night. Developmental Science, 14(3), 481-489. https://doi.org/10.1111/j.14677687.2010.00994.x

LeDoux, J. (2007). The amygdala. In Current Biology (Vol. 17, Issue 20, pp. R868-R874). Elsevier. https://doi.org/10.1016/j.cub.2007.08.005

Lee, E., Kang, J. I., Park, I. H., Kim, J.-J., \& An, S. K. (2008). Is a neutral face really evaluated as being emotionally neutral? Psychiatry Research, 157(1-3), 77-85.

Lee, M. D., \& Wagenmakers, E.-J. (2014). Bayesian cognitive modeling: A practical course. Cambridge university press.

Link, S. (1971). Applying RT deadlines to discrimination reaction time. Psychonomic Science, 25(6), 355-358.

McDermott, J. M., Perez-Edgar, K., \& Fox, N. A. (2007). Variations of the flanker paradigm: Assessing selective attention in young children. Behavior Research Methods, 39(1), 62 70 .

Niendam, T. A., Laird, A. R., Ray, K. L., Dean, Y. M., Glahn, D. C., \& Carter, C. S. (2012). Meta-analytic evidence for a superordinate cognitive control network subserving diverse executive functions. Cognitive, Affective and Behavioral Neuroscience, 12(2), 241-268. https://doi.org/10.3758/s13415-011-0083-5

Norman, D. A., \& Shallice, T. (1986). Attention to Action. In Consciousness and SelfRegulation (pp. 1-18). Springer US. https://doi.org/10.1007/978-1-4757-0629-1_1

Ochsner, K. N., \& Gross, J. J. (2005). The cognitive control of emotion. Trends in Cognitive Sciences, 9(5), 242-249.

Padmala, S., Bauer, A., \& Pessoa, L. (2011). Negative emotion impairs conflict-driven executive control. Frontiers in Psychology, 2, 192.

Peltola, M. J., Leppänen, J. M., Mäki, S., \& Hietanen, J. K. (2009). Emergence of enhanced 
attention to fearful faces between 5 and 7 months of age. Social Cognitive and Affective Neuroscience, 4(2), 134-142. https://doi.org/10.1093/scan/nsn046

Pessoa, L., Kastner, S., \& Ungerleider, L. G. (2002). Attentional control of the processing of neutral and emotional stimuli. Cognitive Brain Research, 15(1), 31-45.

Pessoa, L., Padmala, S., Kenzer, A., \& Bauer, A. (2012). Interactions between cognition and emotion during response inhibition. Emotion, 12(1), 192.

Petersen, I. T., Hoyniak, C. P., McQuillan, M. E., Bates, J. E., \& Staples, A. D. (2016). Measuring the development of inhibitory control: The challenge of heterotypic continuity. Developmental Review, 40, 25-71. https://doi.org/10.1016/j.dr.2016.02.001

Posner, M. I., Rueda, M. R., \& Kanske, P. (2009). Probing the Mechanisms of Attention. In Handbook of Psychophysiology (pp. 410-432). Cambridge University Press. https://doi.org/10.1017/cbo9780511546396.018

R Core Team. (2019). R: A Language and Environment for Statistical Computing. R Foundation for Statistical Computing. https://www.R-project.org/

Scherer, K. R. (1994). Emotion serves to decouple stimulus and response. In P. Ekman \& R. J. Davidson (Eds.), The Nature of Emotion: Fundamental Questions (pp. 127-130). New York: Oxford University Press.

Schmiedek, F., Oberauer, K., Wilhelm, O., Süß, H. M., \& Wittmann, W. W. (2007). Individual Differences in Components of Reaction Time Distributions and Their Relations to Working Memory and Intelligence. Journal of Experimental Psychology: General, 136(3), 414-429. https://doi.org/10.1037/0096-3445.136.3.414

Schönbrodt, F. D., \& Wagenmakers, E. J. (2018). Bayes factor design analysis: Planning for compelling evidence. Psychonomic Bulletin and Review, 25(1), 128-142. https://doi.org/10.3758/s13423-017-1230-y

Schönbrodt, F. D., Wagenmakers, E. J., Zehetleitner, M., \& Perugini, M. (2017). Sequential hypothesis testing with Bayes factors: Efficiently testing mean differences. Psychological Methods, 22(2), 322-339. https://doi.org/10.1037/met0000061

Stroop, J. R. (1935). Studies of interference in serial verbal reactions. Journal of Experimental Psychology, 18(6), 643-662. https://doi.org/10.1037/h0054651

Vaish, A., Grossmann, T., \& Woodward, A. (2008). Not All Emotions Are Created Equal: The Negativity Bias in Social-Emotional Development. Psychological Bulletin, 134(3), 383403. https://doi.org/10.1037/0033-2909.134.3.383

van Doorn, J., van den Bergh, D., Böhm, U., Dablander, F., Derks, K., Draws, T., Etz, A., Evans, N. J., Gronau, Q. F., Haaf, J. M., Hinne, M., Kucharský, Š., Ly, A., Marsman, M., Matzke, D., Gupta, A. R. K. N., Sarafoglou, A., Stefan, A., Voelkel, J. G., \& Wagenmakers, E. J. (2020). The JASP guidelines for conducting and reporting a Bayesian analysis. In Psychonomic Bulletin and Review (pp. 1-14). Springer. https://doi.org/10.3758/s13423-020-01798-5

Van Veen, V., \& Carter, C. S. (2002). The anterior cingulate as a conflict monitor: FMRI and ERP studies. Physiology \& Behavior, 77(4-5), 477-482.

Wagenmakers, E.-J., Marsman, M., Jamil, T., Ly, A., Verhagen, J., Love, J., Selker, R., Gronau, Q. F., Šmíra, M., Epskamp, S., \& others. (2018). Bayesian inference for psychology. Part I: Theoretical advantages and practical ramifications. Psychonomic Bulletin \& Review, 25(1), 35-57.

Weintraub, S., Bauer, P. J., Zelazo, P. D., Wallner-Allen, K., Dikmen, S. S., Heaton, R. K., 
Tulsky, D. S., Slotkin, J., Blitz, D. L., Carlozzi, N. E., \& others. (2013). I. NIH Toolbox Cognition Battery (CB): Introduction and pediatric data. Monographs of the Society for Research in Child Development, 78(4), 1-15.

Zaadnoordijk, L., Buckler, H., Cusack, R., Tsuji, S., \& Bergmann, C. (2021). A Global Perspective on Testing Infants Online: Introducing ManyBabies-AtHome. PsyArXiv. https://doi.org/10.31234/osf.io/cnwh5

Zelazo, P. D., \& Carlson, S. M. (2012). Hot and Cool Executive Function in Childhood and Adolescence: Development and Plasticity. Child Development Perspectives, 6(4), 354360. https://doi.org/10.1111/j.1750-8606.2012.00246.x

Zelazo, P. D., \& Carlson, S. M. (2020). The neurodevelopment of executive function skills: Implications for academic achievement gaps. Psychology \& Neuroscience, 13(3), 273298. https://doi.org/10.1037/pne0000208

Zinchenko, A., Chen, S., \& Zhou, R. (2019). Affective modulation of executive control in early childhood: Evidence from ERPs and a Go/Nogo task. Biological Psychology, 144, 5463.

Zinchenko, A., Kanske, P., Obermeier, C., Schröger, E., \& Kotz, S. A. (2015). Emotion and goal-directed behavior: ERP evidence on cognitive and emotional conflict. Social Cognitive and Affective Neuroscience, 10(11), 1577-1587.

Zinchenko, A., Kanske, P., Obermeier, C., Schröger, E., Villringer, A., \& Kotz, S. A. (2018). Modulation of cognitive and emotional control in age-related mild-to-moderate hearing loss. Frontiers in Neurology, 9, 783.

Zinchenko, A., Obermeier, C., Kanske, P., Schröger, E., \& Kotz, S. A. (2017). Positive emotion impedes emotional but not cognitive conflict processing. Cognitive, Affective and Behavioral Neuroscience, 17(3), 665-677. https://doi.org/10.3758/s13415-017-0504-1

Zinchenko, A., Obermeier, C., Kanske, P., Schröger, E., Villringer, A., \& Kotz, S. A. (2017). The influence of negative emotion on cognitive and emotional control remains intact in aging. Frontiers in Aging Neuroscience, 9, 349. 